\title{
Influência do ciclo hidrológico na dieta e estrutura trófica da ictiofauna do rio Cuiabá, Pantanal Mato-Grossense
}

\author{
Carlos Eduardo Corrêa ${ }^{1}$, Ana Cristina Petry ${ }^{2} \&$ Norma S. Hahn ${ }^{3}$
}

1. Faculdade Eniac, Rua Força Pública, 89, 07012-030 Guarulhos, SP, Brasil. (correa.carloseduardo@yahoo.com.br)

2. Núcleo em Ecologia e Desenvolvimento Sócio-Ambiental de Macaé, Universidade Federal do Rio de Janeiro, Caixa Postal 119331, 27910-970 Macaé, RJ, Brasil.

3. Núcleo de Pesquisas em Limnologia, Ictiologia e Aqüicultura, Universidade Estadual de Maringá, Av. Colombo, 5790, Blocos H90 e G90, Maringá, PR, Brasil.

\begin{abstract}
Influence of the hydrological cycle on the diet and trophic structure of the ichthyofauna from the Cuiabá River, Pantanal of Mato Grosso, Brazil. This paper evaluate the influence of the hydrological cycle on the diet and trophic structure of the ichthyofauna from the Pantanal. Samplings were carried out monthly between March 2000 and February 2001, in the Cuiabá River and the Chacororé Lagoon. Analysis of the stomach contents of 58 species determined eight trophic categories. Species of the piscivore, detritivore and lepidophage trophic categories presented little variation in the intake food items and, therefore, they did not contribute to the variation in the composition of the diet in both environments, independently of the hydrological cycle. The effects of the flood pulse were more pronounced in the numerical abundance and biomass of detritivore and omnivore trophic categories and were depended on the environment for piscivore, invertivore, and insectivore. Apparently, specificity of the food items along with the probable high availability of food resources in the studied environments contributed to the low variation due to the hydrological seasonality in the trophic organization of the ichthyofauna. By the increment of the hydrological connectivity, the flood pulse of the Cuiabá River allows the exchange of organisms throughout the system, thus determining the variability in the structure of the trophic categories.
\end{abstract}

KEYWORDS. Feeding, trophic structure, flood pulse, Neotropical fishes.

RESUMO. Este estudo teve como objetivo avaliar a influência do ciclo hidrológico sobre a dieta e estrutura trófica da ictiofauna no Pantanal. Foram realizadas amostragens mensais entre março de 2000 e fevereiro de 2001, no rio Cuiabá e na lagoa Chacororé. A análise dos conteúdos estomacais de 58 espécies permitiu o reconhecimento de oito categorias tróficas. Peixes das categorias piscívora, detritívora e lepidófaga foram os que apresentaram menor variação na composição da dieta em ambos os ambientes, independente do período hidrológico. Os efeitos do pulso de inundação foram pronunciados na abundância numérica e na biomassa das categorias detritívora e omnívora, enquanto variações nestes parâmetros apresentaram-se relacionadas ao tipo de ambiente para as categorias piscívora, invertívora e insetívora. Os resultados sugeriram que tanto a especialização por alguns itens alimentares quanto a provável elevada disponibilidade de recursos alimentares nos ambientes investigados contribuíram para o fraco efeito do ciclo hidrológico na organização trófica da ictiofauna. Através do aumento da conectividade hidrológica, o pulso de inundação do rio Cuiabá possibilita o deslocamento dos organismos pelo sistema, que deve determinar a variabilidade na estrutura das categorias tróficas.

PALAVRAS-CHAVE. Alimentação, estrutura trófica, pulso de inundação, peixes neotropicais.

Estudos sobre alimentação da ictiofauna permitem reconhecer categorias tróficas distintas e inferir sobre a estrutura e o grau de importância dos diferentes níveis tróficos e sobre as inter-relações entre os seus componentes (Gerking, 1994; AgostinHo et al., 1997). Além de propiciar um campo interessante para a discussão de aspectos teóricos, como a substituição de espécies através dos componentes espacial e temporal (SCHOENER, 1974), estes estudos contribuem para o conhecimento básico da biologia das espécies. WinEMILLER \& JEPSEN (1998) comentam que os peixes são componentes importantes das teias tróficas e que o manejo futuro de estoques pesqueiros em ecossistemas aquáticos tropicais deve ser embasado por um grande conhecimento da ecologia trófica das espécies.

A despeito da ictiofauna de regiões tropicais exibir grupos tróficos especializados, a maioria das espécies apresenta elevada plasticidade alimentar (LoweMcConnell, 1987; ABelha et al., 2001; Hahn et al., 2004), a qual é facilmente verificada pelas constantes variações na dieta. Dessa forma, é muito comum encontrar hábitos alimentares distintos para uma mesma espécie, dependendo do local de captura e das condições hidrológicas no período de amostragem. Levando-se em conta que muitos ambientes de água doce se interconectam, principalmente nos períodos de águas altas, e que os peixes frequentemente transitam de um local para outro, é esperado que estes estejam constantemente sujeitos a uma grande heterogeneidade espaço-temporal na oferta do alimento. De acordo com POWER (2001), ampliar o entendimento sobre as escalas temporal e espacial dos processos envolvidos em teias tróficas constitui-se em um grande desafio, ao mesmo tempo em que a dinâmica e interações entre espécies ainda precisam ser resolvidas.

Na parte mais baixa da bacia do rio Cuiabá existe uma extensa planície, com um complexo sistema de lagoas e várzeas, que compõe a parte superior do Pantanal MatoGrossense, a qual encontra-se sob influência de um regime regular de chuvas. Na bacia do baixo rio Cuiabá foram registradas cerca de 250 espécies de peixes, sendo que, destas, 33 são novas para a ciência (VERíssimo et al., 2005) e sem nenhum registro de dados biológicos ou ecológicos na literatura. Além disso, as características dessa região 
podem mudar em médio ou longo prazo, visto que $300 \mathrm{~km}$ a montante foi formado, em 1999, o reservatório de Manso. Assim, qualquer conhecimento gerado sobre a ictiofauna da região poderá auxiliar em futuras atividades de manejo do estoque pesqueiro.

Considerando um ambiente lótico (rio Cuiabá) e um lêntico (lagoa Chacororé) interligados por um canal e sujeitos ao mesmo regime hidrológico, este trabalho compara a dieta e a estrutura trófica da ictiofauna, com o objetivo de avaliar a influência das variáveis espacial e temporal. Especificamente, procurou-se responder as seguintes questões: (1) o nível hidrológico do rio Cuiabá influencia da mesma maneira a composição da dieta das espécies nos diferentes ambientes estudados? (2) A riqueza de espécies, abundância numérica e a biomassa das categorias tróficas refletem as oscilações do nível hidrológico independentemente do tipo de ambiente (rio ou lagoa)?

\section{MATERIAL E MÉTODOS}

Área de estudo. Neste estudo foram amostrados dois locais no rio Cuiabá (pontos 1 e 2), que se distanciam em cerca de $80 \mathrm{~km}$, e um na lagoa Chacororé (ponto 3), localizada na margem esquerda deste rio, no Estado de Mato Grosso (Fig. 1). Durante o período de águas baixas (maio a outubro), os locais de coleta no rio Cuiabá são caracterizados por um dique marginal elevado (3-5 m) e encostas ocupadas predominantemente por pastagens. Nesse trecho, o rio apresenta cerca de $80 \mathrm{~m}$ de largura, profundidade de aproximadamente $3,5 \mathrm{~m}$ e fundo arenoargiloso, entremeado por pequenas áreas rochosas. Permanentemente conectada ao rio Cuiabá encontra-se a lagoa Chacororé, que apresenta cerca de um metro de profundidade, vegetação do entorno composta por
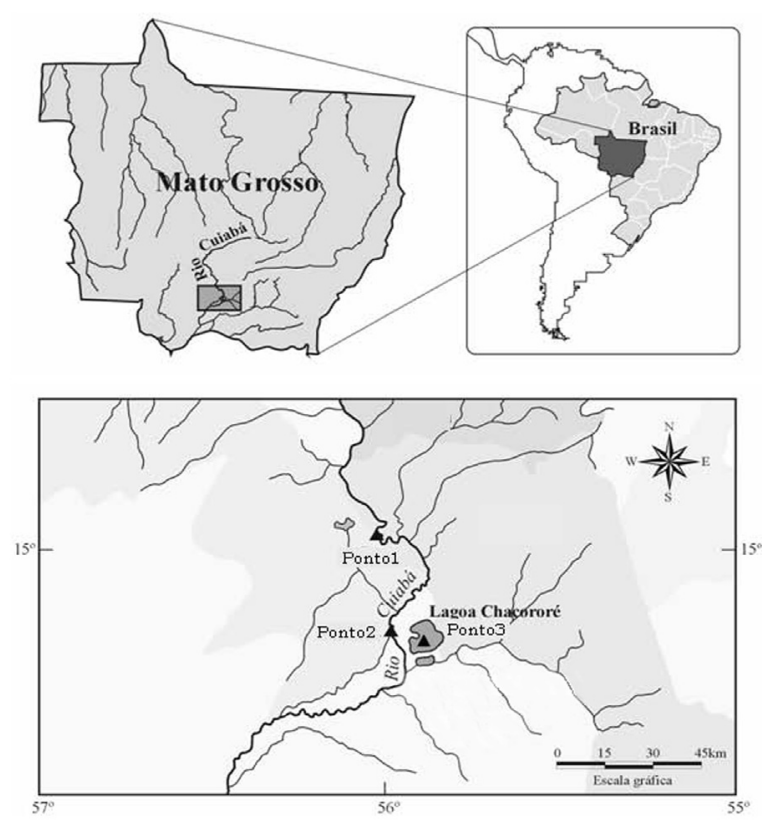

Figura 1. Área de estudo e locais de coleta no rio Cuiabá (1 e 2) e lagoa Chacororé (3), bacia do rio Cuiabá, Mato Grosso. Área clara representa o trecho superior do Pantanal Mato-Grossense. pastagem com manchas de cerrado e densa cobertura de macrófitas aquáticas em uma faixa de $200 \mathrm{~m}$ da porção litorânea.

Amostragem. As amostragens mensais contemplaram os períodos de águas altas (março, abril, novembro e dezembro de 2000, e janeiro e fevereiro de 2001) e baixas (maio a outubro de 2000) (Fig. 2). Os peixes foram amostrados utilizando-se redes de espera simples (esforço padronizado de coleta: 662,60 $\mathrm{m}^{2}$; malhas: 2,4 a $30 \mathrm{~cm}$ entre nós opostos; total de 24 horas: revistas às 8 , 16 e $22 \mathrm{~h}$ ) e arrastos marginais com redes de $20 \mathrm{~m}$ (malha de $1 \mathrm{~cm}$ entre nós). Os indivíduos foram identificados de acordo com BRITSKI et al. (1999) e de cada um foi registrado o comprimento padrão $(\mathrm{cm})$ e o peso total $(\mathrm{g})$. Todos os indivíduos foram eviscerados, tendo os estômagos retirados e fixados em formol $4 \%$ e conservados em álcool $70 \%$. Todas as espécies utilizadas nesse estudo tiveram exemplares testemunho depositados na Coleção de Peixes do Núcleo de Pesquisas em Limnologia, Ictiologia e Aqüicultura (Nupélia), Universidade Estadual de Maringá (UEM).

Análise dos dados. Os conteúdos estomacais foram examinados sob microscópio estereoscópico e óptico. Para determinação quantitativa do alimento, foi utilizado o método volumétrico (HysLOP, 1980), através do qual se estimou o volume de cada item alimentar, obtendo-se a porcentagem (\%V) em relação ao total de todos os conteúdos estomacais para cada espécie, em cada período hidrológico (águas altas e águas baixas) e em cada ambiente (rio e lagoa). Para as análises estatísticas, os itens alimentares foram agrupados em categorias mais amplas. Cada categoria trófica foi determinada em função do predomínio de um determinado tipo de recurso alimentar $(\geq 51 \%$ do volume total da dieta para cada espécie). As espécies cujas dietas não apresentaram predomínio de qualquer recurso alimentar, em particular, foram consideradas omnívoras. Foi investigada a variação espaço-temporal das categorias tróficas através da comparação das amostras obtidas no rio Cuiabá e na lagoa Chacororé, nos períodos de águas altas e baixas.

Para investigar a organização trófica das espécies foi utilizada a análise de correspondência com remoção do efeito do arco (DCA), aplicada aos valores percentuais de volume $(\% \mathrm{~V})$ do conteúdo alimentar, previamente

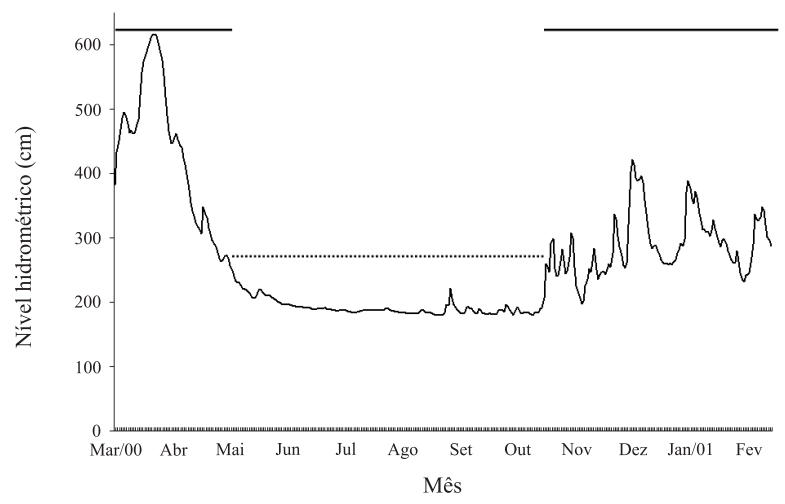

Figura 2. Cotas diárias do nível hidrológico do rio Cuiabá (março/ 00 a fevereiro/01) e indicação dos períodos de águas altas (linha contínua) e baixas (linha tracejada). Dados fornecidos pela Agência Nacional de Águas (ANA). 
transformados em raiz quadrada. Somente os eixos com autovalores superiores a 0,20 foram retidos para interpretação (MATTHEws, 1998). Os padrões observados na análise multivariada (DCA) e outros descritores (riqueza de espécies, abundância numérica e biomassa) foram selecionados para explorar a possível influência do pulso de inundação na estrutura das categorias tróficas nos diferentes ambientes. $\mathrm{O}$ teste de análise de variância (Anova bifatorial) foi aplicado sobre os escores dos eixos da DCA retidos para interpretação e sobre os três descritores, considerando como fatores o período hidrológico (águas altas e baixas) e o tipo de ambiente (rio e lagoa). As análises multivariadas e os testes de médias foram efetuados através dos softwares PC-ORD (McCune \& MefFord, 1997) e Statistica 7.1 (StatSoft InC., 2005), respectivamente. Os dados de abundância numérica e de biomassa foram log-transformados $\left(\log _{10} \mathrm{x}+1\right)$ para que os pressupostos de normalidade e homocedasticidade fossem atendidos. O Teste de Tukey foi aplicado sempre que diferenças significativas $(P<$ $0,05)$ foram detectadas entre as médias. Para todas as análises foram selecionadas somente as espécies que tiveram número de estômagos analisados $\geq 4$.

\section{RESULTADOS}

Um total de 2.090 indivíduos, pertencentes a 58 espécies foi capturado durante as amostragens e, de modo geral, a ictiofauna foi caracterizada como de pequeno porte $(57 \%<15 \mathrm{~cm} \mathrm{CP})$ (Tab. I). Com base na análise da dieta as espécies foram organizadas em oito categorias tróficas: algívora, detritívora, herbívora, insetívora, invertívora, omnívora, lepidófaga e piscívora (Tab. I). Não foi observada qualquer segregação trófica na ordenação das espécies entre os períodos hidrológicos e/ou ambientes, exceto pela clara distinção da categoria lepidófaga no rio (Fig. 3). Por outro lado, a ordenação dos recursos alimentares em relação aos padrões de similaridade alimentar das espécies resultou na formação de um contínuo no espaço formado pelos dois primeiros eixos da DCA (Fig. 4). Juntos, estes eixos explicaram $45 \%$ da variância da matriz original dos valores de conteúdo alimentar (Fig. 4). No eixo DCA 1 (autovalor =0,66), as espécies piscívoras posicionaram-se nos maiores escores e estiveram positivamente correlacionadas com o eixo, enquanto que as detritívoras e algívoras posicionaramse nos menores escores e estiveram negativamente correlacionadas com o eixo. As demais categorias posicionaram-se nos escores intermediários e foram influenciadas pela baixa dominância dos recursos alimentares na dieta. No eixo DCA 2 (autovalor $=0,43$ ), as espécies lepidófagas alcançaram os maiores escores e se segregaram das demais (Fig. 4). O resultado da ANOVA aplicada aos escores dos eixos DCA1 e DCA2 (Fig. 5) confirmou a inspeção das figuras 3 e 4 . A ausência de interações significativas permitiu que a influência de cada fator (período hidrológico e tipo de ambiente) fosse avaliada independentemente (Tab. II). Diferenças significativas entre os períodos de águas altas e baixas e entre o rio e a lagoa não foram detectadas para ambos os eixos, DCA1 e DCA2 (Fig. 5; Tab. II).

Dois aspectos na avaliação da variação espaçotemporal das categorias tróficas foram: o consumo de peixes por espécies tipicamente lepidófagas durante as águas altas na lagoa e a grande proporção de espécies piscívoras e detritívoras que não alteraram suas dietas, a despeito das variações hidrológicas marcantes (Tab. I).

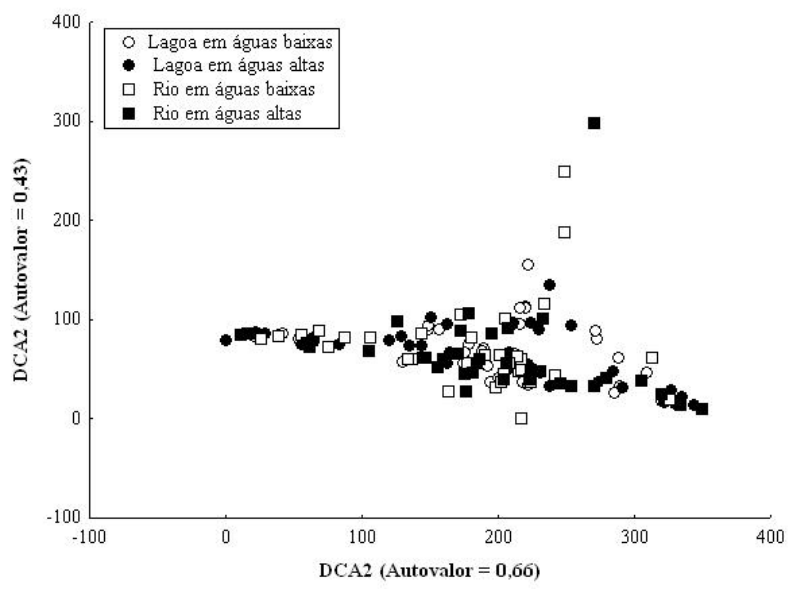

Figura 3. Ordenação da ictiofauna de acordo com o recurso alimentar consumido na bacia do rio Cuiabá, Estado de Mato Grosso entre março/00 e fevereiro/01.

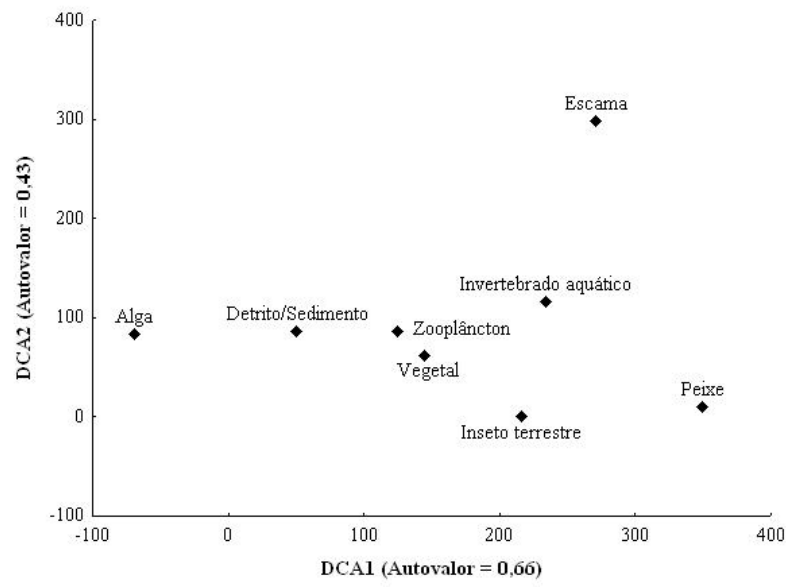

Figura 4. Ordenação dos recursos alimentares utilizados pela ictiofauna na bacia do rio Cuiabá, Estado de Mato Grosso entre março/00 e fevereiro/01.

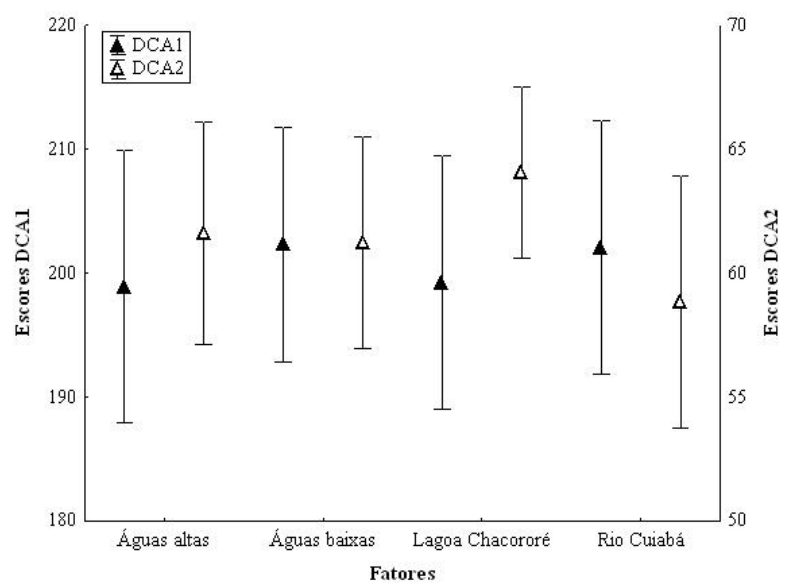

Figura 5. Valores médios (erro-padrão) dos escores dos dois primeiros eixos da DCA, considerando o ciclo hidrológico (águas altas e águas baixas) e ambientes (rio Cuiabá e lagoa Chacororé) na bacia do rio Cuiabá, Estado de Mato Grosso entre março/00 e fevereiro/01. 
Tabela I. Espécies de peixes amostradas, número de estômagos analisados (N) e comprimento padrão dos indivíduos (CP; cm) capturados no rio Cuiabá e lagoa Chacororé, Estado de Mato Grosso (março/00 a fevereiro/01) (Alg, algívora; Det, detritívora; Her, herbívora; Ins, insetívora; Inv, invertívora; Lep, lepidófaga; Omn, omnívora; Pis, piscívora).

\begin{tabular}{|c|c|c|c|c|c|c|}
\hline \multirow{2}{*}{ Táxons } & \multirow{2}{*}{$\mathrm{N}$} & \multirow{2}{*}{$\mathrm{CP}$} & \multicolumn{2}{|c|}{ Águas altas } & \multicolumn{2}{|c|}{ Águas Baixas } \\
\hline & & & Rio & Lagoa & Rio & Lagoa \\
\hline \multicolumn{7}{|l|}{ PRISTIGASTERIDAE } \\
\hline Pellona flavipinnis (Valenciennes, 1836) & 9 & $9,0-31,5$ & & Pis & & Pis \\
\hline \multicolumn{7}{|l|}{ CURIMATIDAE } \\
\hline Curimatella dorsalis (Eigenmann \& Eigenmann, 1889) & 10 & $4,4-11,3$ & Det & Det & Det & Det \\
\hline Potamorhina squamoralevis (Braga \& Azpelicueta, 1983) & 18 & $17,5-22,9$ & Det & Det & Det & Det \\
\hline Psectrogaster curviventris Eigenmann \& Kennedy, 1903 & 20 & $10,5-15,0$ & Her & Her & Her & Det \\
\hline Steindachnerina conspersa (Holmberg, 1891) & 4 & $5,7-10,2$ & & Det & Det & \\
\hline S. nigrotaenia (Boulenger, 1902) & 11 & $3,6-7,3$ & & Det & Det & \\
\hline \multicolumn{7}{|l|}{ PROCHILODONTIDAE } \\
\hline \multicolumn{7}{|l|}{ ANOSTOMIDAE } \\
\hline Abramites hypselonotus (Günther, 1868) & 13 & $5,2-10,5$ & Her & & Det & \\
\hline \multicolumn{7}{|l|}{ CRENUCHIDAE } \\
\hline $\begin{array}{l}\text { Characidium aff. zebra Eigenmann, } 1909 \\
\text { HEMIODONTIDAE }\end{array}$ & 11 & $1,8-3,0$ & & & Det & Inv \\
\hline $\begin{array}{l}\text { Hemiodus orthonops Eigenmann \& Kennedy, } 1903 \\
\text { GASTEROPELECIDAE }\end{array}$ & 22 & $8,8-19,5$ & & Alg & Det & \\
\hline $\begin{array}{l}\text { Thoracocharax stellatus (Kner, 1858) } \\
\text { ERYTHRINIDAE }\end{array}$ & 118 & $1,8-5,0$ & Ins & Ins & Ins & Ins \\
\hline Hoplias aff. malabaricus (Bloch, 1794) & 15 & $1,8-27,0$ & & Pis & & Pis \\
\hline CYNODONTIDAE & & & & & & \\
\hline Rhaphiodon vulpinus Agassiz, 1829 & 12 & $12,4-57,0$ & Pis & Pis & Pis & Pis \\
\hline CHARACIDAE & & & & & & \\
\hline Aphyocharax anisitsi Eigenmann \& Kennedy, 1903 & 63 & $1,5-3,2$ & & In $v$ & & Ins \\
\hline A. dentatus Eigenmann \& Kennedy, 1903 & 144 & $1,8-5,7$ & Pis & $\mathrm{Pis}$ & Pis & Pis \\
\hline Astyanax abramis (Jenyns, 1842) & 15 & $4,0-7,4$ & Her & In $v$ & Ins & Ins \\
\hline Astyanax sp. 1 & 4 & $3,4-4,0$ & Ins & & Ins & \\
\hline Bryconamericus exodon Eigenmann, 1907 & 112 & $1,6-3,8$ & $\mathrm{Omn}$ & & In $v$ & Ins \\
\hline Bryconops melanurus (Bloch, 1794) & 6 & $1,7-5,0$ & Ins & & Ins & \\
\hline Bryconamericus sp. 1 & 15 & $2,5-3,7$ & & & Ins & \\
\hline Clupeacharax anchoveoides Pearson, 1924 & 4 & $4,0-5,4$ & In $v$ & & Ins & \\
\hline Galeocharax humeralis (Valenciennes, 1834) & 35 & $4,1-22,0$ & Pis & Inv & Pis & \\
\hline Hemigrammus marginatus Ellis, 1911 & 72 & $1,4-4,0$ & In $v$ & In $v$ & Pis & Ins \\
\hline Hyphessobrycon eques (Steindachner, 1882) & 12 & $1,7-2,2$ & & & & In $v$ \\
\hline Moenkhausia dichroura (Kner, 1858) & 42 & $1,8-5,2$ & $\mathrm{Omn}$ & Her & $\mathrm{Omn}$ & Ins \\
\hline Mylossoma duriventre (Cuvier, 1818) & 21 & $10,7-17,5$ & Her & & Her & \\
\hline Piabarchus analis (Eigenmann, 1914) & 5 & $2,4-2,9$ & & & Ins & \\
\hline Piaractus mesopotamicus (Holmberg, 1887) & 4 & $33,5-45,5$ & Her & & Her & \\
\hline Poptella paraguayensis (Eigenmann, 1907) & 18 & $3,0-3,7$ & Ins & Omn & & $\mathrm{Omn}$ \\
\hline Psellogrammus kennedyi (Eigenmann, 1903) & 12 & $2,3-3,7$ & & $\mathrm{Omn}$ & & $\mathrm{Omn}$ \\
\hline Pygocentrus nattereri Kner, 1858 & 100 & $10,0-32,0$ & Pis & Pis & Pis & Pis \\
\hline Roeboides descalvadensis Fowler, 1932 & 84 & $2,0-6,4$ & $\mathrm{Omn}$ & Lep & Lep & Inv \\
\hline R. microlepis (Reinhardt, 1851) & 22 & $9,2-19,5$ & Pis & & Pis & Inv \\
\hline R. affinis (Günther, 1868) & 79 & $5,0-10,5$ & Lep & In $v$ & Pis & Lep \\
\hline Salminus brasiliensis (Cuvier, 1816) & 12 & $11,0-43,5$ & Pis & $\mathrm{Pis}$ & Pis & Pis \\
\hline Serrasalmus maculatus Kner, 1858 & 12 & $7,2-15,6$ & & Pis & Pis & $\mathrm{Pis}$ \\
\hline S. marginatus Valenciennes, 1837 & 21 & $7,3-265$ & Pis & Pis & Pis & \\
\hline Tetragonopterus argenteus Cuvier, 1816 & 54 & $1,9-9,6$ & Pis & Her & $\mathrm{Omn}$ & $\mathrm{Omn}$ \\
\hline Triportheus pantanensis Malabarba, 2004 & 52 & $2,2-13,3$ & Her & $\mathrm{Alg}$ & Ins & Her \\
\hline T. nematurus (Kner, 1858) & 113 & $1,8-17,5$ & Her & $\mathrm{Omn}$ & Ins & $\mathrm{Omn}$ \\
\hline LORICARIIDAE & & & & & & \\
\hline Pterygoplichthys ambrosettii (Holmberg, 1893) & 17 & $16-34,0$ & & Omn & & Det \\
\hline HEPTAPTERIDAE & & & & & & \\
\hline Pimelodella gracilis (Valenciennes, 1835) & 98 & $3,2-7,6$ & In $v$ & In $v$ & Inv & In $v$ \\
\hline P. mucosa Eigenmann \& Ward, 1907 & 16 & $5,2-7,3$ & & & Her & In $v$ \\
\hline PIMELODIDAE & & & & & & \\
\hline Iheringichthys labrosus (Lütken, 1874) & 19 & $1,8-17,4$ & $\mathrm{Omn}$ & & Inv & Inv \\
\hline Pimelodus cf. argenteus Perugia, 1891 & 155 & $2,4-18,5$ & Pis & Pis & $\mathrm{Omn}$ & $\mathrm{Omn}$ \\
\hline P. maculatus Lacepède, 1803 & 44 & $3,6-20,1$ & & Omn & Pis & $\mathrm{Omn}$ \\
\hline DORADIDAE & & & & & & \\
\hline Pterodoras granulosus (Valenciennes, 1821) & 41 & $18,5-37,5$ & Her & Her & Her & \\
\hline $\begin{array}{l}\text { Trachydoras paraguayensis (Eigenmann \& Ward, 1907) } \\
\text { AUCHENIPTERIDAE }\end{array}$ & 12 & $6,4-9,5$ & In $v$ & $\mathrm{Omn}$ & $\mathrm{Omn}$ & In $v$ \\
\hline Ageneiosus ucayalensis Castelnau, 1855 & 4 & $3,0-23,5$ & Pis & & & \\
\hline Auchenipterus nigripinnis (Boulenger, 1895) & 39 & $1,7-20,0$ & Ins & Inv & Ins & Ins \\
\hline A. osteomystax (Miranda Ribeiro, 1918) & 25 & $13,5-20,5$ & & In $v$ & In $v$ & Ins \\
\hline Entomocorus benjamini Eigenmann, 1917 & 4 & $2,6-3,1$ & $\mathrm{Omn}$ & & & \\
\hline STERNOPYGIDAE & & & & & & \\
\hline Eigenmannia trilineata López \& Castello, 1966 & 12 & $0,8-15,7$ & Inv & In $v$ & Inv & Inv \\
\hline E. virescens (Valenciennes, 1836) & 104 & $0,7-23,8$ & In $v$ & Ins & Inv & In $v$ \\
\hline SCIAENIDAE & & & & & & \\
\hline $\begin{array}{l}\text { Plagioscion ternetzi Boulenger, } 1895 \\
\text { CICHLIDAE }\end{array}$ & 62 & $1,5-33,0$ & Pis & Pis & Pis & Pis \\
\hline Bujurquina vittata (Heckel, 1840) & 4 & $2,2-3,0$ & & & & $\mathrm{Omn}$ \\
\hline Gymnogeophagus balzanii (Perugia, 1891) & 17 & $2,2-8,5$ & & Pis & & Inv \\
\hline
\end{tabular}


Tabela II. Resultados da ANOVA bifatorial aplicada aos escores dos eixos 1 e 2 da Análise de Correspondência com remoção do efeito do arco (DCA) e descritores da estrutura trófica da ictiofauna do rio Cuiabá e lagoa Chacororé, Mato Grosso (março/00 a fevereiro/01) (* diferenças significativas, $P<0,05)$

\begin{tabular}{|c|c|c|c|c|c|c|c|c|c|c|c|c|}
\hline & \multicolumn{3}{|c|}{ Período } & & \multicolumn{3}{|c|}{ Ambiente } & & \multicolumn{3}{|c|}{ Período x Ambiente } & \\
\hline & GL & $\mathrm{F}$ & $P$ & & GL & $\mathrm{F}$ & $P$ & & GL & $\mathrm{F}$ & $P$ & \\
\hline DCA 1 & 1 & 0,06 & 0,81 & & 1 & 0,05 & 0,83 & & 1 & 0,35 & 0,55 & \\
\hline DCA 2 & 1 & 0,01 & 0,97 & & 1 & 0,71 & 0,40 & & 1 & 0,01 & 0,98 & \\
\hline \multicolumn{13}{|c|}{ Riqueza de Espécies } \\
\hline Detritívora & 1 & 12,37 & $<0,01$ & $*$ & 1 & 0,00 & 1,00 & & 1 & 1,89 & 0,18 & \\
\hline Herbívora & 1 & 36,84 & $<0,01$ & $*$ & 1 & 13,58 & $<0,01$ & $*$ & 1 & 0,75 & 0,39 & \\
\hline Insetívora & 1 & 90,85 & $<0,01$ & $*$ & 1 & 0,05 & 0,03 & $*$ & 1 & 29,83 & $<0,01$ & $*$ \\
\hline Invertívora & 1 & 5,66 & 0,02 & $*$ & 1 & 47,91 & $<0,01$ & $*$ & 1 & 13,52 & $<0,01$ & $*$ \\
\hline Omnívora & 1 & 1,87 & 0,18 & & 1 & 6,82 & 0,02 & $*$ & 1 & 1,25 & 0,27 & \\
\hline Piscívora & 1 & 9,14 & $<0,01$ & $*$ & 1 & 2,29 & 0,14 & & 1 & 49,78 & $<0,01$ & $*$ \\
\hline \multicolumn{13}{|c|}{ Abundância numérica $\left(\log _{10} \mathrm{x}+1\right)$} \\
\hline Detritívora & 1 & 15,05 & $<0,01$ & $*$ & 1 & 0,78 & 0,38 & & 1 & 0,34 & 0,36 & \\
\hline Herbívora & 1 & 1,82 & 0,19 & & 1 & 0,34 & 0,56 & & 1 & 0,40 & 0,53 & \\
\hline Insetívora & 1 & 53,03 & $<0,01$ & $*$ & 1 & 0,34 & 0,56 & & 1 & 39,42 & $<0,01$ & $*$ \\
\hline Invertívora & 1 & 1,07 & 0,31 & & 1 & 24,54 & $<0,01$ & $*$ & 1 & 34,74 & $<0,01$ & $*$ \\
\hline Omnívora & 1 & 7,93 & $<0,01$ & $*$ & 1 & 0,00 & 0,99 & & 1 & 0,04 & 0,85 & \\
\hline Piscívora & 1 & 0,15 & 0,70 & & 1 & 22,63 & $<0,01$ & $*$ & 1 & 0,24 & 0,02 & $*$ \\
\hline \multicolumn{13}{|c|}{ Abundância em biomassa $\left(\log _{10} x+1\right)$} \\
\hline Detritívora & 1 & 12,10 & $<0,01$ & $*$ & 1 & 5,19 & 0,03 & $*$ & 1 & 0,28 & 0,60 & \\
\hline Herbívora & 1 & 0,59 & 0,45 & & 1 & 19,13 & $<0,01$ & $*$ & 1 & 0,01 & 0,91 & \\
\hline Insetívora & 1 & 86,84 & $<0,01$ & $*$ & 1 & 2,14 & 0,15 & & 1 & 21,15 & $<0,01$ & $*$ \\
\hline Invertívora & 1 & 5,21 & $<0,03$ & $*$ & 1 & 12,94 & $<0,01$ & $*$ & 1 & 22,61 & $<0,01$ & $*$ \\
\hline Omnívora & 1 & 0,48 & 0,49 & & 1 & 20,99 & $<0,01$ & $*$ & 1 & 9,44 & $<0,01$ & $*$ \\
\hline Piscívora & 1 & 3,97 & 0,06 & & 1 & 8,55 & $<0,01$ & $*$ & 1 & 0,04 & 0,84 & \\
\hline
\end{tabular}

As tendências de relativa similaridade na organização trófica das espécies entre os períodos hidrológicos e ambientes não foi verificada para os descritores da estrutura das categorias tróficas (Fig. 6). A ausência de interações significativas indicada pela ANOVA aplicada sobre os valores mensais de riqueza de espécies, abundância numérica e biomassa das categorias detritívora e herbívora permitiu que a influência do período hidrológico e do ambiente fosse avaliada independentemente (Tab. I). Para as demais categorias, o efeito do período hidrológico do rio Cuiabá dependeu do tipo de ambiente considerado. Com exceção das categorias algívora (registrada apenas no período de águas altas, na lagoa), e lepidófaga (registrada apenas no período de águas altas, no rio), as demais foram ricas em espécies e comuns aos períodos hidrológicos e ambientes.

Piscívora e invertívora foram representadas por um número maior de espécies (média \pm desvio padrão $9 \pm 1,45$; $5 \pm 1,91$, respectivamente), enquanto que para a algívora e lepidófaga este número foi de no máximo duas espécies, especialmente na lagoa Chacororé. As demais categorias apresentaram em média três espécies (Fig. 6). A interação entre as fontes de variação (período hidrológico $\mathrm{x}$ tipo de ambiente) determinou as maiores diferenças observadas para as categorias tróficas, exceto para detritívora e omnívora (Fig. 6, Tab. II). A riqueza de espécies foi significativamente maior, no período de águas baixas, para as insetívoras e invertívoras na lagoa e, no período de águas altas, para as herbívoras e piscívoras no rio Cuiabá. Com relação à riqueza de espécies, detritívora foi significativamente maior apenas no período de águas baixas e omnívora apenas na lagoa (Fig. 6, Tab. II).

Juntas, as categorias invertívora, piscívora, omnívora e insetívora compreenderam cerca de $80 \%$ da abundância numérica total (Fig. 6). Interações significativas entre os fatores na abundância numérica de insetívora, invertívora e piscívora revelaram que, para essas, o efeito do nível hidrológico foi dependente do tipo de ambiente (Tab. II). Na lagoa Chacororé, as insetívoras foram significativamente mais abundantes nas águas baixas e as invertívoras e piscívoras nas águas altas, sendo que, para as últimas, o número de indivíduos superou em duas vezes aquele registrado no rio (Fig. 6). Efeitos pronunciados e diferenciados do período hidrológico foram detectados para as detritívoras e omnívoras, que foram mais numerosas nos períodos de águas baixas e altas, respectivamente. Espécies piscívoras, detritívoras e herbívoras, especialistas em seu recurso alimentar, compuseram cerca de $90 \%$ da biomassa (Fig. 6). Para as omnívoras, invertívoras e insetívoras, menos representativas em biomassa, foram detectadas interações significativas entre os fatores (Tab. II). A biomassa de invertívoras e omnívoras na lagoa foi significativamente superior durante as águas altas (60 e $50 \%$ do total registrado, respectivamente), enquanto que, para as insetívoras, a biomassa foi superior durante as águas baixas (65\% do total registrado) (Fig. 6; Tab. II). Os efeitos pronunciados do período hidrológico sobre a biomassa foram detectados apenas para as detritívoras, significativamente mais abundantes nas águas baixas, independentemente do ambiente. Um efeito do ambiente foi verificado para as piscívoras e detritívoras, que foram significativamente mais abundantes em biomassa na lagoa, e para as herbívoras, significativamente mais abundante em biomassa no rio, esta última representada especialmente por Pterodoras granulosus (Valenciennes, 1821) e Mylossoma duriventre (Cuvier, 1818). Em função da ausência de replicação espaço-temporal, as categorias algívora e lepidófaga foram excluídas das análises estatísticas dos descritores da estrutura trófica. 
Riqueza de espécies
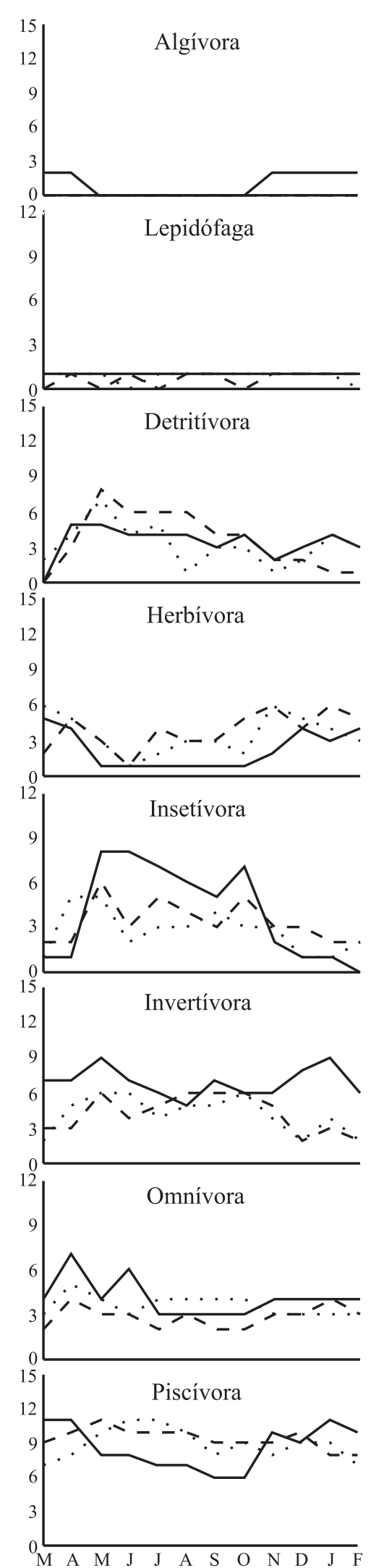

Abundância
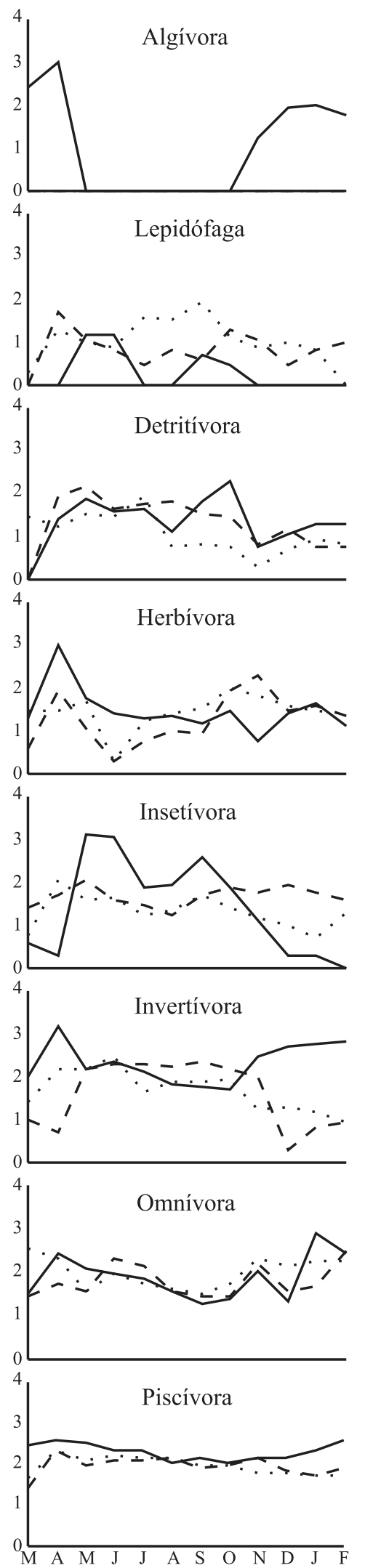

Biomassa

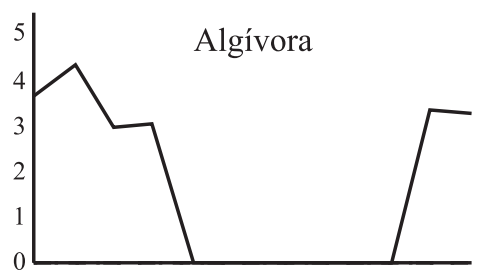

Lepidófaga
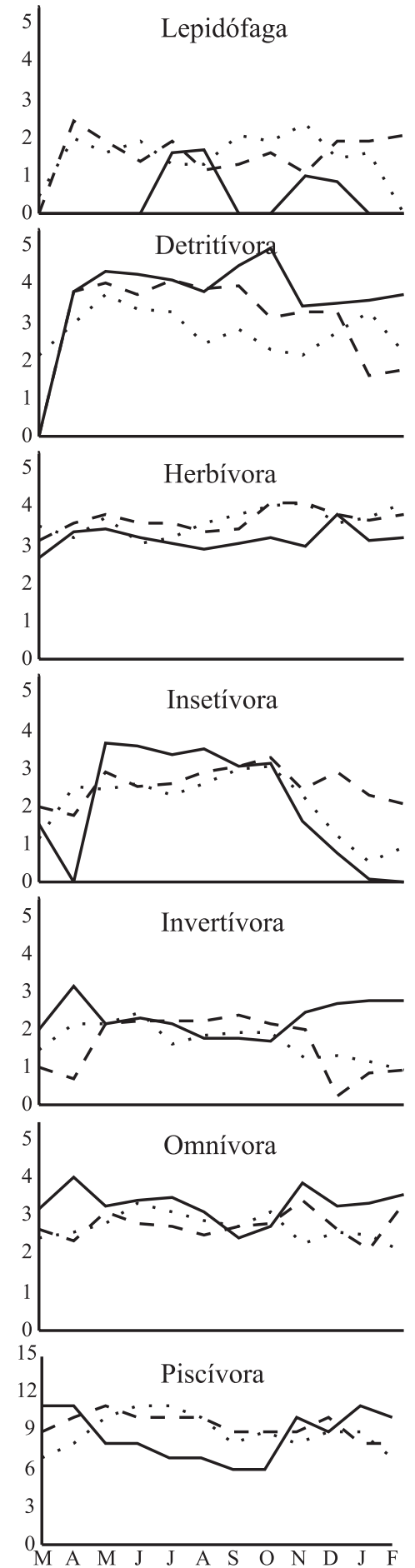

Figura 6. Riqueza de espécies, abundância numérica $\left(\log _{10} x+1\right)$ e biomassa $\left(\log _{10} x+1\right)$ das categorias tróficas da ictiofauna no rio Cuiabá (1, linha pontilhada; 2, linha tracejada) e lagoa Chacororé (linha contínua), Mato Grosso. Período de águas altas - março a abril/00 e novembro/00 a fevereiro/01; período de águas baixas - maio a outubro/00. 


\section{DISCUSSÃO}

Mundialmente, planícies de inundação são consideradas reservatórios da diversidade ictiofaunística regional uma vez que funcionam como locais de alimentação e crescimento para espécies migradoras de longas distâncias e hábitat permanente para as sedentárias, geralmente de menor porte (Welcomme, 1979; Bayley, 1995; Agostinho \& Zalewski, 1996; Bailly et al., 2008). Em sistemas de planície de inundação, as lagoas marginais são reconhecidas como hábitat preferencial de peixes de pequeno porte, principalmente devido à grande heterogeneidade estrutural, disponibilidade de recursos e conexão sazonal com o restante do sistema durante as cheias (Petry et al., 2003). Estas generalidades foram também confirmadas no presente estudo.

Os peixes da bacia do rio Cuiabá exploraram vários tipos de recursos alimentares, uma vez que algas, vegetais superiores, detritos, insetos, invertebrados e peixes foram registrados nos conteúdos estomacais de espécies capturadas tanto em águas altas quanto em águas baixas, no rio Cuiabá e na lagoa Chacororé. Assim, a técnica de ordenação revelou que uma proporção considerável das espécies, principalmente aquelas com adaptações tróficas (ex. detritívoras, piscívoras e herbívoras), não apresentou alterações espaço-temporais relevantes no consumo do alimento. De fato, as espécies que foram estritamente piscívoras, apresentam padrões morfológicos adaptados ao forrageamento de outros peixes, tais como uma ampla abertura bucal (Hoplias aff. malabaricus (Bloch, 1794), Salminus brasiliensis (Cuvier, 1816), Aphyocharax dentatus Eigenmann \& Kennedy, 1903 e Plagioscion ternetzi Boulenger, 1985), dentes cortantes (Pygocentrus nattereri Kner, 1858, Serrasalmus maculatus Kner, 1858 e S. marginatus Valenciennes,1816) e caninos desenvolvidos (Rhaphiodon vulpinus Agassiz, 1829) (Winemiller \& Kelso-Winemiller, 1993; Almeida et al., 1997; Mittelbach \& Persson, 1998). Dentre as detritívoras, Prochilodus lineatus (Valenciennes, 1837) e todos os representantes de Curimatidae, possuem adaptações no canal alimentar para o processamento do detrito (Bowen, 1983; Fugi et al., 2001), tais como estômago químico e mecânico e intestino longo (Bowen, 1983). Três das cinco espécies consideradas aqui como exclusivamente detritívoras - Curimatella dorsalis (Eigenmann \& Eigenmann, 1889), Steindachnerina conspersa (Holmberg, 1891) e P. lineatus - exibiram o mesmo hábito alimentar na planície de inundação do rio Miranda (RESENDE, 2000), outro afluente importante da margem esquerda do alto rio Paraguai. Adicionalmente, as poucas espécies exclusivamente herbívoras apresentam adaptações para processar matéria vegetal e foram aqui representadas por Mylossoma duriventre (Cuvier, 1818) e Piaractus mesopotamicus (Holmberg, 1887), as quais apresentam dentes molariformes para triturar frutos e matéria vegetal (BRITSKI et al., 1999; HAHN et al., 2004). No rio São Francisco, esta categoria trófica foi a mais representativa em abundância numérica e biomassa (ALviM \& Peret, 2004). Apesar dos dentes exteriorizados adaptados para arrancar escamas, as duas espécies de Roeboides, reconhecidas como predominantemente lepidófagas (SAZIMA \& MACHADO, 1982; HAHN et al., 2000; NovAKOWsKI et al., 2004), apresentaram variação na dieta independente do ambiente considerado, consumindo, em alguns casos, uma quantidade maior de invertebrados aquáticos e pedaços de peixes do que de escamas.

$\mathrm{O}$ fato de muitas das espécies estudadas não terem alterado sua dieta no tempo e no espaço parece contradizer o padrão esperado para ambientes aquáticos continentais tropicais, onde a elevada plasticidade trófica exibida pelos peixes é amplamente documentada (Welcomme, 1979; Lowe-McConNell, 1987; Gerking, 1994), especialmente em sistemas temporalmente instáveis, sujeitos à forte sazonalidade hidrológica (Winemiller, 1990; Poff \& Allan, 1995; Hahn et al., 2004). Contudo, outros autores reportaram tendências similares às encontradas neste estudo, ou seja, a ocorrência de um elevado número de espécies especialistas em relação às generalistas (MérONA et al., 2001; Novakowski et al., 2008). Para A. dentatus, $H$. aff. malabaricus e $P$. ternetzi, a ingestão de peixes por indivíduos menores de $2 \mathrm{~cm}$ sugere que a piscivoria tem início nos primeiros meses de vida, corroborando padrões determinados para peixes de ambientes temperados (Mittelbach \& Persson, 1998). A reduzida alteração espaço-temporal na dieta da maior parte das espécies pode indicar uma elevada abundância dos recursos nos ambientes investigados. No entanto, o reduzido número de estômagos analisados para algumas delas sugere cautela nessas inferências.

A técnica de ordenação mostrou segregação das categorias tróficas mais especializadas (piscívora, detritívora e lepidófaga) em relação às demais, com exceção da herbívora, que foi influenciada por espécies que utilizaram vegetais superiores como alimento principal apenas de forma facultativa. Além disso, as algívoras e herbívoras foram mais abundantes apenas no final do período de águas altas, na lagoa e no rio, respectivamente. Excetuando as piscívoras, que se destacaram em abundância numérica e biomassa, os resultados encontrados refletiram as relações negativas comumente observadas entre abundância e porte dos indivíduos. Bryconamericus exodon Eigenmann, 1907, por exemplo, é uma espécie de pequeno porte (não ultrapassou $3,8 \mathrm{~cm}$ ) que, em função de seus hábitos alimentares diversificados, foi caracterizada como invertívora, insetívora e omnívora, dependendo do período hidrológico e do ambiente. De modo geral, as espécies omnívoras são bem sucedidas na colonização de ambientes que variam hidrologicamente e são expressivas no Pantanal MatoGrossense, destacando-se tanto em abundância numérica como em biomassa (Resende, 2000).

A constância na abundância numérica de determinada categoria sugere que o recurso alimentar preferencial esteja disponível em quantidade suficiente para evitar interações negativas, como a competição ou a busca por locais mais adequados para a alimentação, uma vez que a conexão permanente entre a lagoa e o rio permite o trânsito dos organismos (NovAKOWSKI et al., 2008). Assim, o predomínio e a constância na lagoa de consumidores dos níveis superiores (piscívoras e invertívoras) nas águas altas e de níveis basais (detritívoras) nas águas baixas devem estar associados às elevadas diversidade e abundância de peixes-presa, ao farto suprimento de invertebrados aquáticos e à disponibilidade de detrito orgânico (característico dos ambientes produtivos associados às planícies de inundação) (SOARES et al., 1986; RESENDE, 2000; HAHN et al., 2004; PoulLly et al., 2004). A elevada abundância de indivíduos na categoria invertívora, juntamente com a 
detritívora, corroborou as observações feitas em outros sistemas hidrologicamente íntegros, como o rio Mamoré (Poullly et al., 2004), e diferiu daqueles fortemente alterados pela regulação da vazão hidrológica exercida por barragens, tais como o alto rio Paraná (HAHN et al., 2004). Tendências similares para a biomassa, no entanto, não foram verificadas, uma vez que a maior parte das espécies dessas categorias não ultrapassou $5 \mathrm{~cm}$ de comprimento.

Conclui-se, dessa forma, que a influência exercida pelo pulso de inundação do rio Cuiabá não foi suficiente para alterar a organização trófica da ictiofauna, independentemente do ambiente investigado. Dois fatores, não mutuamente exclusivos, podem explicar os padrões encontrados: o primeiro se relaciona ao grau de especialização trófica do consumidor, cujo espectro é limitado por especialização morfológica e/ou comportamental; e o segundo, à disponibilidade dos recursos alimentares no espaço e no tempo, que parece não ter sido modificada sazonalmente. Por outro lado, a estrutura das categorias tróficas, excetuando a herbívora e a detritívora, refletiu as oscilações do nível hidrológico do rio Cuiabá. No entanto, a intensidade do efeito do pulso de inundação sobre a riqueza de espécies, densidade numérica e biomassa esteve na dependência do tipo de ambiente. Dessa forma, o pulso de inundação do rio Cuiabá parece maximizar os efeitos espaciais da conectividade hidrológica ao possibilitar o deslocamento dos consumidores pelo sistema.

Agradecimentos. Agradecemos ao Núcleo de Pesquisas em Limnologia, Ictiologia e Aquiicultura e ao Programa de PósGraduação em Ecologia de Ambientes Aquáticos Continentais da Universidade Estadual de Maringá pelo apoio financeiro e suporte logístico; à Coordenação de Aperfeiçoamento de Pessoal de Nível Superior pela bolsa de doutorado ao primeiro autor e a L. C. Gomes pela correção do Abstract. Ao CNPq pela bolsa de produtividade concedida a N. S. H.

\section{REFERÊNCIAS BIBLIOGRÁFICAS}

Abelha, M. C. F.; Agostinho, A. A. \& Goulart, E. 2001. Plasticidade trófica em peixes de água doce. Acta Scientiarum 23(2):425-434.

Agostinho, A. A. \& Zalewski, M. 1996. A planície alagável do alto rio Paraná: importância e preservação. Maringá, EDUEM. 100p.

Agostinho, A. A.; Hahn, N. S.; Gomes, L. C. \& Bini, L. M. 1997. Estrutura trófica. In: Vazzoler, A. E. A. M.; Agostinho, A. A. \& Hahn, N. S. eds. A planície de inundação do alto rio Paraná: aspectos físicos, biológicos e socioeconômicos. Maringá, EDUEM. p.229-248.

Almeida, V. L. L.; Hahn, N. S. \& Vazzoler, A. E. A. M. 1997. Feeding patterns in five predatory fishes of the high Paraná River floodplain (PR, Brazil). Ecology of Freshwater Fish 6(3):123-133.

Alvim, M. C. C. \& Peret, A. C. 2004. Food resources sustaining the fish fauna in a section of the upper São Francisco river in Três Marias, MG, Brazil. Brazilian Journal of Biology 64:195-202.

Bailly, D.; Agostinho, A. A. \& Suzuki, H. I. 2008. Influence of the flood regime on the reproduction of fish species with different reproductive strategies in the Cuiabá River, upper Pantanal, Brazil. River Research \& Applications 24:1218-1229.

BAyley, P. B. 1995. Understanding large river-floodplain ecosystems. BioScience 45(3):153-158.

Bowen, S. H. 1983. Detritivory in neotropical fishes communities. Environmental Biology of Fishes 9(2):137-144.

Britski, H. A.; Silimon, K. Z. S. \& Lopes, B. S. 1999. Peixes do Pantanal - manual de identificação. Brasília, EMBRAPA. 184p.
Fugi, R.; Agostinho, A. A. \& Hahn, N. S. 2001. Trophic morphology of five benthic-feeding fish species of a tropical floodplain. Revista Brasileira de Biologia 61(1):27-33.

Gerking, S. D. 1994. Feeding ecology of fish. San Diego, Academic. $416 \mathrm{p}$.

Hahn, N. S.; Fugi, R.; Lourero-CripPa, V. E.; Peretti, D. \& Russo, M. R. 2004. Trophic structure of the fish fauna In: Agostinho, A. A.; Rodrigues, L.; Gomes, L. C.; Thomaz, S. M. \& Miranda, L. E. eds. Structure and funcioning of the Paraná River and its floodpalin. Maringá, EDUEM. p.139-143.

Hahn, N. S.; Pavanelli, C. S \& OKada, E. K. 2000. Dental development and ontogenetic diet shifts of Roeboides paranensis (Osteichthyes, Characinae) in pools of the rio Paraná floodplain (State of Paraná, Brazil). Revista Brasileira de Biologia 60(1):93-99.

Hyslop, E. P. 1980. Stomach contents analysis, a review of methods and their application. Journal of Fish Biology 17:411-429.

Lowe-McConnell, R. H. 1987. Ecological studies in tropical fish communities. Cambridge, Cambridge University. 382p.

Matthews, W. J. 1998. Patterns in freshwater fish ecology. New York, Chapman \& Hall. 756p.

McCune, B. \& MefFord, M. J. 1997. Multivariate analysis of ecological data, version 3.0. Oregon, MjM Software Design.

Mérona, B.; Santos, G. M. \& Almeida, R. G. 2001. Short term effects of Tucuruí Dam (Amazonia, Brazil) on the trophic organization of fish communities. Environmental Biology of Fishes 60:375-392.

Mittelbach, G. \& Persson, L. 1998. The ontogeny of piscivory and its ecological consequences. Canadian Journal of Fisheries and Aquatic Sciences 55:1454-1465.

Novakowski, G. C.; Fugi, R. \& Hahn, N. S. 2004. Diet and dental development of three species of Roeboides (Characiformes: Characidae). Neotropical Ichthyology 2(3):157-162.

Novakowski, G. C.; HAHn, N. S. \& Fugi, R. 2008. Diet seasonality and food overlap of the fish assemblage in a pantanal pond. Neotropical Ichthyology 6(4):567-576.

Petry, A. C.; Agostinho, A. A. \& Gomes, L. C. 2003. Fish assemblages of tropical floodplain lagoons: exploring the role of connectivity in a dry year. Neotropical Ichthyology 1(2):111-119.

Poff, N. L. \& Allan, J. D. 1995. Functional organization of stream fish assemblages in relation to hydrological variability. Ecology 76(2):606-627

Poullly, M.; Yunoki, T.; Rosales, C. \& Torres, L. 2004. Trophic structure of fish assemblages from Mamoré river floodplain lakes (Bolivia). Ecology of Freshwater Fish 13:245-257.

Power, M. E. 2001. Prey exchange between a stream and its forested watershed elevates preator densities in both habitats Proceedings of the National Academy of Sciences 98:14,15.

Resende, E. K. 2000. Trophic structure of fish assemblage in the lower Miranda river, Pantanal, Mato Grosso do Sul State, Brazil. Revista Brasileira de Biologia 60(3):89-403.

Sazima, I. \& Machado, F. A. 1982. Hábitos e comportamento de Roeboides prognathus, um peixe lepidófago (Osteichthyes, Characoidei). Boletim de Zoologia da Universidade de São Paulo 7:37-56.

Schoener, T. W. 1974. Resource partitioning in ecological communities. Science 185:27-38

Sonres, M. G. M.; Almeida, R. G. \& Junk, W. J. 1986. The trophic status of the fish fauna in Lago Camaleão, a macrophyte dominated floodplain lake in the middle Amazon. Amazoniana 9:511-526.

StatSoft, Inc. 2005. Statistica (data analysis software system), version 7.1. www.statsoft.com.

Veríssimo, S.; Pavanelli, C. S.; Britski, E. \& Moreira, M. M. M. 2005. Fish, Manso Reservoir region of influence, Rio Paraguai basin, Mato Grosso State, Brazil. Check List (UNESP) 1(1):1-9.

Welcome, R. L. 1979. Fisheries ecology of floodplain rivers. London, Longman. $317 \mathrm{p}$.

WinEMILLER, K. O. 1990. Spatial and temporal variation in tropical fish trophic networks. Ecological Monographs 60:331-367.

Winemiller, K. O. \& JePsen, D. B. 1998. Effects of seasonality and fish movement on tropical river food webs. Journal of Fish Biology 53:267-296.

Winemiller, K. O. \&. Kelso-Winemiller, L. C. 1993. Predatory response of piranhas to alternative prey. National Geographic Research 9:344-357. 